\title{
A comparison of machine learning regression techniques for LiDAR-derived estimation of forest variables
}

\author{
J. García-Gutiérrez, F. Martínez-Álvarez, A. Troncoso, J.C. Riquelme
}

Keywords:

LiDAR

Machine learning

Regression

Remote sensing

A B S T R A C T

\begin{abstract}
Light Detection and Ranging (LiDAR) is a remote sensor able to extract three-dimensional information. Environmental models in forest areas have been benefited by the use of LiDAR-derived information in the last years. A multiple linear regression (MLR) with previous stepwise feature selection is the most common method in the literature to develop those models. MLR defines the relation between the set of field measurements and the statistics extracted from a LiDAR flight. Machine learning has emerged as a suitable tool to improve classic stepwise MLR results on LiDAR. Unfortunately, few studies have been proposed to compare the quality of the multiple machine learning approaches. This paper presents a comparison between the classic MLR-based methodology and regression techniques in machine learning (neural networks, support vector machines, nearest neighbour, ensembles such as random forests) with special emphasis on regression trees. The selected techniques are applied to real LiDAR data from two areas in the province of Lugo (Galizia, Spain). The results confirm that classic MLR is outperformed by machine learning techniques and concretely, our experiments suggest that Support Vector Regression with Gaussian kernels statistically outperforms the rest of the techniques.
\end{abstract}

\section{Introduction}

Light Detection and Ranging (LiDAR) is a remote laser-based technology which differs from optic sensors in its ability to determine heights of objects. LiDAR is able to measure the distance from the source to an object or surface providing not only $x-y$ position, but also the coordinate $z$ for every impact. The distance to the object is determined by measuring the time between the pulse emission and detection of the reflected signal taking into account the position of the emitter.

LiDAR sensors have transformed the way to perform many important tasks for the natural environment. The work previously done with expensive or not always-feasible fieldwork has partially been replaced by the processing of airborne LiDAR point cloud (initial product obtained from a LiDAR flight). Although the development of digital elevation models has traditionally been the main use of LiDAR [1,2], applications for other purposes can also be found in the literature. Thus, research work often aims to the extraction of descriptive variables from LiDAR and their use to develop products related to urban or environmental mapping and forest management. For those tasks, machine learning and, more precisely supervised learning, is usually the selected tool in the form of both classification (used in most urban or environmental mapping approaches) and regression (most common in estimation of biophysical variables).

Regarding classification, we can find techniques such as Support Vector Machines or Random Forests (RF) applied to LiDAR (isolated or fused with other information sources, e.g., multispectral images) for the development of forest inventories [3,4] or fuel models [5]. But even when classification has been also important for LiDAR, researchers have specially focused on deriving variables related to the LiDAR's ability to extract vertical information and then, worked on establishing relations with field measurements. Hence, regression techniques have been paid a greater attention to improve empirical models. Following this philosophy, LiDAR can currently be found for different tasks such as estimation of biomass in forest areas [6] or prediction of building ages [7].

In the case of forest LiDAR-derived models, we can observe multiple linear regression (MLR) has usually been the main tool for the estimation of parameters regressed from LiDAR statistics. The main advantage of using this type of methodology is the simplicity and clarity of the resulting model. In contrast, the selected method also has some drawbacks: this process provides a set of highly correlated predictors with little physical justification [8] and, as a 
parametric technique, it is only recommended when assumptions such as normality, homocedasticity, independence and linearity are met [9].

With the previous in mind, it is important to outline that methodologies to develop regression models between field-work data and LiDAR are being reviewed [10]. As a consequence, machine learning non-parametric regression techniques have started to be applied with success.

Our aim in this work is to compare the most well-known regression techniques of machine learning in a common framework. Thus, we can establish a ranking when they are applied to forest variable estimation to help environmental researchers for the selection of the most suitable technique for their needs. The different techniques have been tested and statistically validated on two LiDAR datasets from two different areas of the province of Lugo (Galizia, Spain).

The rest of the paper is organized as follows. Section 2 provides a general review of the state of the art and Section 3 shows a description of the LiDAR data used in this work. The methodology is presented in Section 4. The results achieved, their statistical validation and the main findings are shown in Section 5. Finally, Section 6 is devoted to summarize the conclusions and to discuss future lines of work.

\section{Related work}

Researchers have already explored advanced regression techniques for forest variables estimation and recent literature provides examples which show their suitability in comparison with MLR. Thus, Chen and Hay [11] used Support Vector Regression (SVR) to estimate biophysical features of vegetation from LiDAR data and multispectral images outperforming classical stepwise regression. Their results were confirmed by Jachowski et al. [12] although SVR was only applied to multispectral images in this case.

Decision trees in the form of ensembles such as RF have been applied with good results. Thus, Latifi et al. showed [13] how they could be used for biomass estimation and outperform classical stepwise regression after an evolutionary feature selection. Even without the evolutionary feature selection, similar results have been also reported by other researchers [14]. Moreover, they were not only used for onshore biomass estimation but also employed to model and predict seafloor standing stocks [15] and for large datasets where they showed a performance as good as that of smaller ones [16].

In addition to SVR and RF as the most extended machine learning regression techniques recently published in the literature, other techniques have also been explored. Thus, Zhao et al. [17] provided a comparison between Gaussian Processes (GP) and stepwise MLR where the first ones clearly improved the results after a set of composite features were extracted from a LiDAR point cloud. Hudak et al. [18] applied nearest neighbours to extract relations between LiDAR and fieldwork for several vegetation species at plot level.

Although machine learning seems to be suitable to extract meaningful information from LiDAR, few studies have been provided to compare the quality of the regressions obtained by different sets of techniques. For instance, Gleason and Im [19] showed a partial comparison of methods where SVR outperformed RF and Li et al. [20] established a deep comparison among machine learning techniques where SVMs and boosted decision trees obtained the best results though simple ordinary least squares approach performed just as well as any advanced machine learning method. Recently Gagliasso et al. [21] examined the predictive performance of linear regression, geographic weighted regression, gradient nearest neighbour, most similar neighbour, RF imputation, and k-Nearest
Neighbour (kNN) to estimate biomass and basal area. A combination of ground inventory plots, LiDAR data, satellite imagery, and climate data was analyzed, and the root mean square error (RMSE) and bias were calculated to test the different methods. In this case, results showed that for biomass prediction, the $\mathrm{kNN}(k=5)$ had the lowest RMSE and the least amount of bias. Although a statistical validation of results is commonly required for a comparison of machine learning techniques [22], no statistical study was provided for either of the previous works which would have been desirable to generalize their conclusions.

Stojanova et al. [23] performed a comparison of the results of different types of regression trees, in particular, isolated trees and ensembles such as RF. The results confirmed the use of ensembles improved the performance when the estimation of forest variables was carried out. Although a deep statistical validation was applied to the results, no regression technique from other families such as kNN or SVR was compared with the regression trees.

After studying the possible improvements of the recent bibliography, we carried out a comparison of the most well-known regression techniques of machine learning in a common framework. Then we established a ranking when they were applied to the estimation of forest variables after being tested and statistically validated on two LiDAR datasets from two different areas of the province of Lugo (Galizia, Spain).

\section{Materials}

\subsection{Study sites}

Aerial LiDAR data in two forest areas in the northwest part of the Iberian Peninsula (Fig. 1) were used for this study (more details about both areas can be found in Goncalves-Seco et al. [24] and Gonzalez-Ferreiro et al. [25], respectively).

The first study area (hereafter site A) was located in Trabada, concretely in the municipality of Vilapena (Galicia, NW Spain; boundaries 644800; 4806600 and 645800; 4810600 UTM). Eucalyptus globulus stands, with low intensity silvicultural treatments and the presence of tall shrubs, dominated the forest type.

The second study area (hereafter site B) was also located in Galicia (NW Spain), in the municipality of Guitiriz, and covered about $36 \mathrm{~km}^{2}$ of Pinus radiata forests (boundaries 586315; 4783000 and $595102 ; 4787130$ UTM). P. radiata was the main forest type in this area and its stands were also characterized by low-intensity silvicultural treatments and by the presence of tall shrubs.

\subsection{Field data}

Field data from the two study sites were collected to obtain the dependent variables for the regressions in this work. Thus, 39 instances (one per training plot in the study site) were located and measured on site A. On site B, a similar process was carried out for a total of 54 plots. The plots were selected to represent the existing range of ages, stand sizes, and densities in the studied forests.

For site A and B, the dry weight of the biomass fractions of each tree was estimated using the equations for E. globulus in Galicia reported by Dieguez-Aranda et al. [26]. In order to define the dependent variables, the field measurements (heights and diameters) and the estimated dry weight of the biomass fractions were used to calculate the following stand variables in each plot: stand crown biomass $\left(W_{c r}\right)$, stand stem biomass $\left(W_{s t}\right)$, and stand aboveground biomass $\left(W_{a b g}\right)$.

In the case of site $\mathrm{B}$, the field measurements (heights and diameters) and the estimated volumes and dry weight of the biomass fractions helped to estimate the following additional 

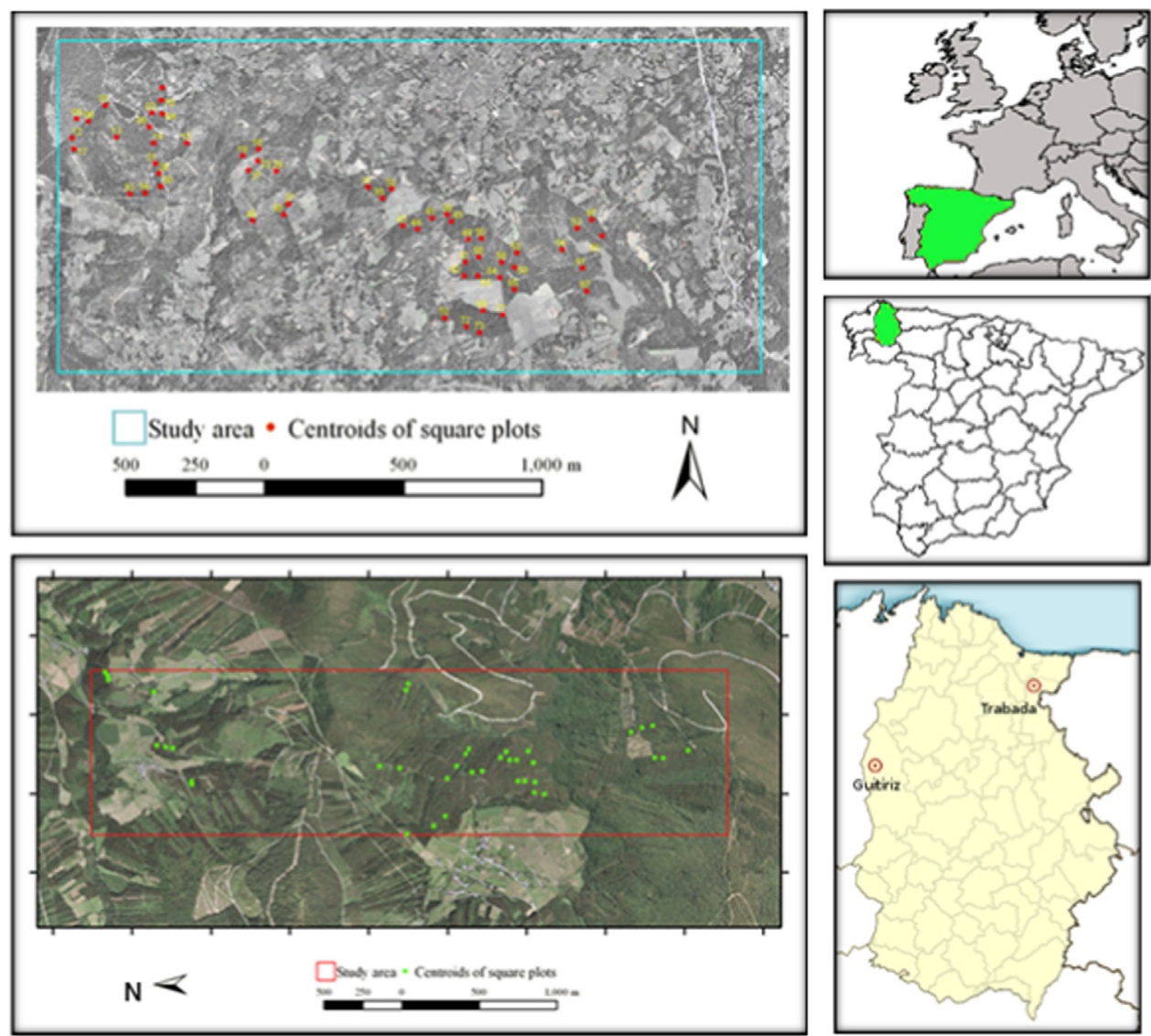

Fig. 1. Study sites located in the province of Lugo (NW of Spain). Top: study site of Guitiriz. Bottom: study site of Trabada.

stand variables in each plot: stand basal area $(G)$, dominant height $\left(H_{d}\right)$, mean height $\left(H_{m}\right)$, and stand volume $(V)$.

\subsection{LiDAR data}

The LiDAR data from site A were acquired in November 2004. The first and last return pulses were registered. The whole study area was flown over 18 strips and each strip was flown over three times, which gave an average measurement density of about 4 pulses $\mathrm{m}^{-2}$. The LiDAR data for site B were acquired in September 2007. An averaged laser pulse density of 8 pulses $\mathrm{m}^{-2}$ was obtained. In order to obtain two additional different resolutions, an artificial reduction based on a random selection of LiDAR pulses in a grid cell of $1 \mathrm{~m}^{2}$ was carried out for each flight. They resulted in two new LiDAR datasets with a pulse density of 0.5 pulses [25].

Intensity values in both study sites were normalized to eliminate the influence of path height variations [3]. Filtering, interpolation, and the development of Digital Terrain and Canopy Models (DTM/ DCM) were performed by FUSION software [27]. This software also provided the variables related to the height and return intensity distributions within the limits of the field plots in the four datasets (original and reduced data from study sites A and B). Table 1 shows the complete set of metrics and the corresponding abbreviations used in this paper.

From field data and the statistics obtained from LiDAR we built 60 datasets. Each database was composed of the 48 independent variables $\left(C O V E R_{F P}\right.$ and RETURNS in Table 1 together with the rest of the variables calculated for intensity and heights) extracted from LiDAR data and a dependent variable (fieldwork-derived forest variable), given a study site and a resolution. This procedure gave a total of 20 datasets ( 4 and 8 forest variables for site A and B, respectively, multiplied by 2 different resolutions). The remaining
Table 1

Statistics extracted from the LiDAR flights' heights and intensities used as independent variables for the regression models.

\begin{tabular}{llll}
\hline Description & Abbreviation & Description & Abbreviation \\
\hline Percentage of first & & 25th percentile & P25 \\
returns over $2 \mathrm{~m}$ & COVER_FP & 50th percentile & P50 \\
Number of returns above 2 $\mathrm{m}$ & RETURNS & 75th percentile & P75 \\
Minimum & MIN & 5th percentile & P05 \\
Maximum & MAX & 10th percentile & P10 \\
Mean & MEAN & 20th percentile & P20 \\
Mode & MODE & 30th percentile & P30 \\
Standard deviation & SD & 40th percentile & P40 \\
Variance & V & 60th percentile & P60 \\
Interquartile distance & ID & 70th percentile & P70 \\
Skewness & SKW & 80th percentile & P80 \\
Kurtosis & KURT & 90th percentile & P90 \\
Average absolute deviation & AAD & 95th percentile & P95 \\
\hline
\end{tabular}

40 datasets were obtained using two types of feature transformation (power and exponential) commonly used in the literature [25] on the previous 20 .

\section{Regression techniques}

\subsection{Regression trees}

Decision trees based on If-Then rules are one of the most popular methods used in machine learning for classification since they offer results that can be easily interpreted. Thus, this approach obtains ordered sets of If-Then rules for prediction that produces understandable models [28]. Regression trees thus offer 
to LiDAR scientist the power of a non-parametric regression technique, which is frequently higher than MLR's when parametric conditions are not met, with an open-box (understandable) model which is often required by environmental users with no expertise in machine learning.

In this work, eight kinds of novel regression tree structures have been applied to forest variables prediction from LIDAR data. What makes them different from each other are the regression models considered in the leaf nodes. Specifically, we consider the following models:

Model MEAN-LM: This model is a constant linear model. This constant value is the mean of the target variable for all instances of the training set that reach the leaf.

Model MED-LM: This model is also a constant linear model, but the constant value is the median instead of the mean.

Model LM: Linear model in which the coefficients are computed by minimizing the mean square error.

Model MDL-LM: Linear model in which the coefficients are computed based on a minimum description length principle.

Model RLM: This model is a reduced linear model. Once the linear model is obtained by the least square method, it is simplified as in M5' algorithm [29]. In particular, a greedy search is used to determine which variables of the linear function can be removed by minimizing the estimated error.

Model $k N N-L M$ : This model is a local non-linear model, based on the $k$-NN algorithm. In this case, the forecasting is divided into two steps clearly differentiated. In a first step, the $k$ nearest neighbours are searched for between the instances of the training set that reach the leaf, and secondly, the mean of the target variable of the $k$ nearest neighbours is computed. Thus, the previously computed mean is the prediction.

Model WkNN-LM: This model is also a local non-linear model based on the weighted $k$ nearest neighbours. The prediction is the weighted mean of the target variable of the $k$ nearest neighbours and the weights $w_{i}$ are given by a Gaussian kernel:

$w_{i}=\exp \left(\frac{d\left(x_{i}, n\left(x_{i}\right)\right)}{\sigma}\right)$

where $d$ is the Euclidean distance between a point $x_{i}$ and its $i$-th nearest neighbour $n\left(x_{i}\right), \sigma$ is the width of the Gaussian kernel and $\exp (\cdot)$ is the exponential function.

Model LWLR: This model is a local linear model based on a locally weighted linear regression, in which the coefficients are computed by minimizing the weighted mean square error and the weights are given by a distance function.

Summarizing, six linear models (five global and one local) and two non-linear local models are considered to construct regression trees. In general, the linear models present some limitations since when models in leaves are too simple, some functions cannot be approximated. On the other hand, global models have a lower computational cost than local models, since the latter have to be rebuilt for each point of the test set. In this work, the package CORElearn [30] (available in software R) has been used to generate the different regression trees described above.

\subsection{Other techniques}

With the goal of comparing the results of the regression trees above with several families of machine learning techniques applied to LiDAR data for estimation of forest variables, we selected the most extended machine learning algorithms in the literature such as support vector machines for regression, artificial neural networks, nearest neighbour rule and ensembles of regression trees among others. Namely, we included in the study the algorithms implemented in the open source software for data mining WEKA [31]: M5P (another implementation of the M5' regression tree),
SMOreg (SVR with polynomial, SMO-p, and Gaussian, SMO-g, kernels), LinearRegression (classical MLR), MultilayerPerceptron (MLP, a type of neural network), Gaussian processes (with Gaussian, GP-g, and polynomial, GP-p, kernels) and IBk (an implementation of $\mathrm{kNN}$ ). On the other hand, we developed an ad hoc RF which consists in replacing its random trees by M5P trees. This change was necessary because the original implementation in WEKA only allows its use for classification and not for regression.

\subsection{Comparison framework}

The regression trees and the M5P, SMO-p, SMO-g, MLP, IBk, GPg, GP-p, MLR and RF techniques described in Sections 4.1 and 4.2, respectively, were applied to the 60 testing datasets.

The comparison was defined from the coefficients of correlation, $R$, as was done in recent bibliography [17]. Also we included an analysis of the results in terms of root mean square error (RMSE). In our case, the coefficients obtained in a process of LeaveOne-Out Cross-Validation (LOOCV) on each dataset since the number of instances was too small for a more adequate $n$-fold cross-validation. The best and mean values in 100 executions were recorded for each technique and dataset in order to obtain robust results (independent from parameterization).

All algorithms were used after applying a preprocessing phase of normalization and elimination of missing values. Moreover, feature selection to avoid the Hughes phenomenon [32] was also applied. The feature selection based on the well-known Correlation Feature Selection (CFS) filter from Weka was used. Since most techniques implement a feature selection phase in their own building (e.g., regression trees, support vector machines and RF) we decided to carry out each experiment with and without the CFS feature selection. Thus, we could obtain the best result regardless of whether the algorithms could obtain better results with their own feature selection.

As we said, all the considered algorithms were tested on every dataset 100 times using different configuration setups. Moreover, each tuple 〈〈configuration setup, algorithm, dataset〉〉 was carried out twice: one with feature selection (CFS) and one without. Then, we selected the best of the two to be part of the 100 partial results.

Regarding each algorithm setups, they were defined as random values for each parameter needed to execute the algorithm. Thus, a random value between 1 and 20 was selected for the number of neighbours in IBk, kNN-LM and WkNN-LM models and a $\sigma$ parameter in the interval [1, number of instances] was also used for WkNN-LM. In the case of the support vector machines, the parameter $C$ was defined in the interval $[1,20]$. In addition, SVM-g uses a Gaussian kernel which depends on a parameter $g$ defined in the interval [0.01,1]. For SVM-p, which uses a polynomial kernel, the exponent for the polynomial was set up with a value between 1 and 3. Our RF only has two parameters: the number of trees and the number of predictors used per tree. They were in the intervals $[1,20]$ and $[1,100]$, respectively. Finally, the MLP performance is controlled by three parameters: number of hidden layers, momentum and learning rate. They were in the intervals $[1,20]$, $[0.01,1]$ and $[0.01,1]$, respectively.

\section{Results}

This section provides the results obtained by the application of all the examined algorithms described in Section 4. Results for all the algorithms in terms of maximum and mean $R$ and improvement of RMSE regarding the worst technique for each dataset are reported in Section 5. Tests to determine whether the results can be considered statistically different or not have been carried out in Section 5.2. Discussion of the results obtained can be found in Section 5.3 . 


\subsection{Performance of the algorithms}

Fig. 2 shows the influence of each statistic on the automatic selections made by the CFS algorithm. Refer to Table 2 to check how variables are mapped into the columns, where Column \#1 is associated with the leftmost column in the figure, and Column \#48 with the rightmost one. In this sense, a zero value (black) means that the variable was not selected, whereas a one value (white) means just the opposite, that is, the variable was selected.

Figs. 3 and 4 illustrate the maximum and mean of $R$. For visual purposes, Figs. 5 and 6 do not provide RMSE but the percentage of improvement regarding the RMSE of the worst regressor for each dataset when best and mean RMSE are studied, respectively. This representation has been chosen since RMSE is not a dimensionless measure which involves very different values if we are dealing with forest variables such as canopy biomass $(\mathrm{kg} / \mathrm{ha})$ or basal area $\left(\mathrm{m}^{2}\right)$. Each row of the figures identifies one dataset whereas the columns identify each of the seventeen algorithms. Refer to Table 3 to check how algorithms are mapped into the columns where Column \#1 is associated with the leftmost column in the four figures, and Column \#17 with the rightmost one. In addition, due to the high number of datasets studied, we provide

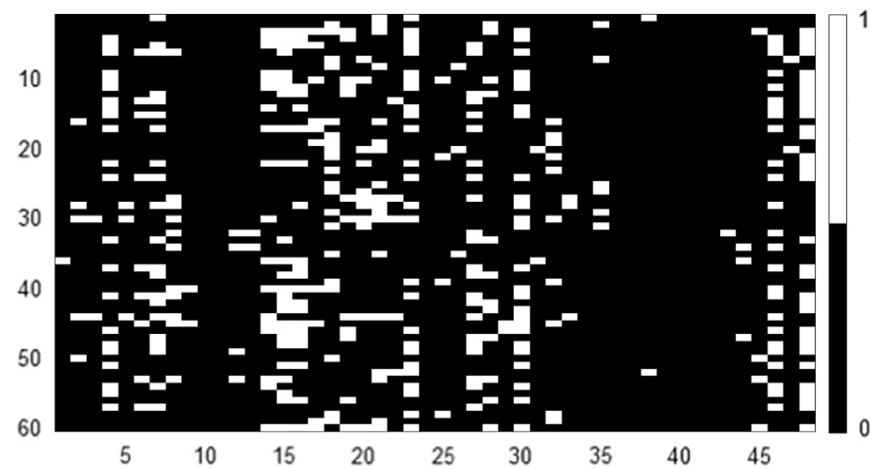

Fig. 2. Selection of LiDAR-derived features by the CFS method through the 60 datasets. A value of 1 means a feature was selected; otherwise, the value is 0 .

Table 2

Correspondence between features and columns for Fig. 2. Variable names starting by $\mathrm{H}$ and I were obtained from height and intensity distributions, respectively.

\begin{tabular}{llll}
\hline Column & Variable & Column & Variable \\
\hline$\# 1$ & COVER_FP & $\# 25$ & IAAD \\
$\# 2$ & HAAD & $\# 26$ & IID \\
$\# 3$ & HID & $\# 27$ & IKURT \\
$\# 4$ & HKURT & $\# 28$ & IMAX \\
$\# 5$ & HMAX & $\# 29$ & IMEAN \\
$\# 6$ & HMEAN & $\# 30$ & IMIN \\
$\# 7$ & HMIN & $\# 31$ & IMODE \\
$\# 8$ & HMODE & $\# 32$ & IP05 \\
$\# 9$ & HP05 & $\# 33$ & IP10 \\
$\# 10$ & HP10 & $\# 34$ & IP20 \\
$\# 11$ & HP20 & $\# 35$ & IP25 \\
$\# 12$ & HP25 & $\# 36$ & IP30 \\
$\# 13$ & HP30 & $\# 37$ & IP40 \\
$\# 14$ & HP40 & $\# 38$ & IP50 \\
$\# 15$ & HP50 & $\# 39$ & IP60 \\
$\# 16$ & HP60 & $\# 40$ & IP70 \\
$\# 17$ & HP70 & $\# 41$ & IP75 \\
$\# 18$ & HP75 & $\# 42$ & IP80 \\
$\# 19$ & HP80 & $\# 43$ & IP90 \\
$\# 20$ & HP90 & $\# 44$ & IP95 \\
$\# 21$ & HP95 & $\# 45$ & ISD \\
$\# 22$ & HSD & $\# 46$ & ISKW \\
$\# 23$ & HSKW & $\# 47$ & IV \\
$\# 24$ & HV & $\# 48$ & RETURNS \\
\hline & & &
\end{tabular}

Tables 4 and 5 which summarize the main statistics for every technique regarding $R$ and the percentage of improvement of RMSE, respectively.

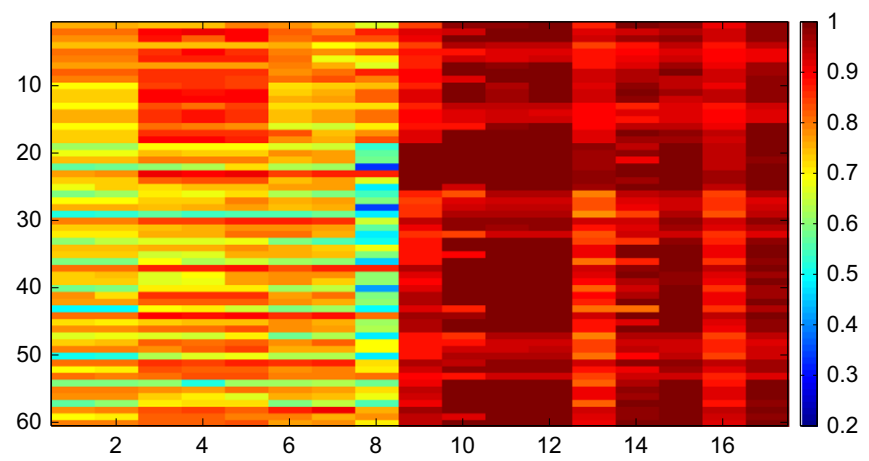

Fig. 3. Maximum $R$ values after 100 executions for the 60 datasets each.

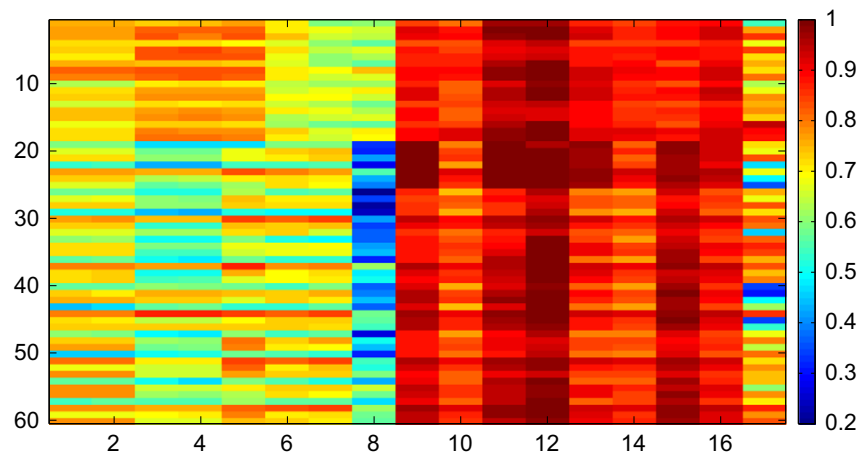

Fig. 4. Mean $R$ values after 100 executions for the 60 datasets each.

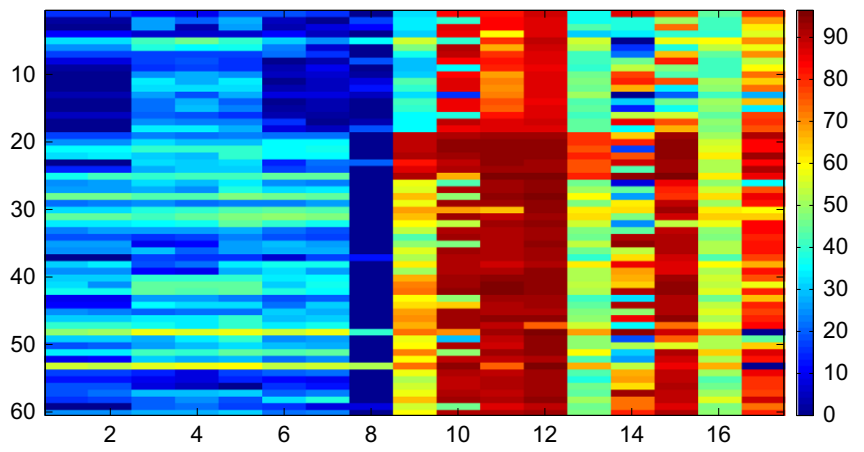

Fig. 5. Maximum percentage of improvement regarding the best RMSE reached by the worst competitor after 100 executions for the 60 datasets.

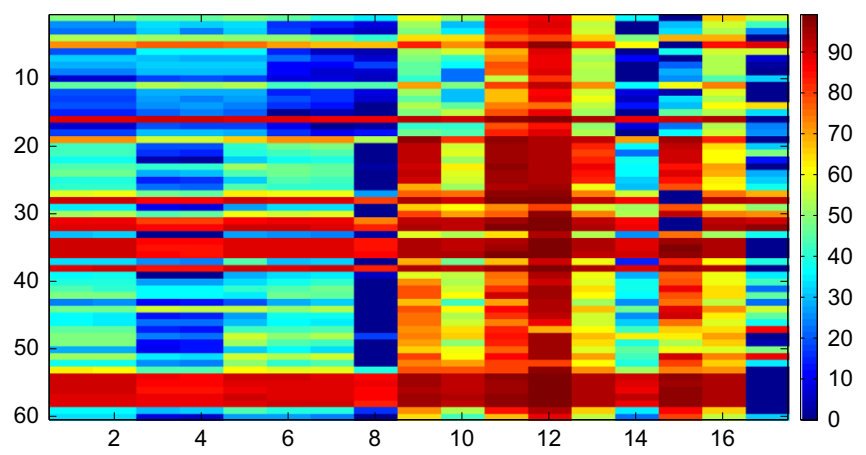

Fig. 6. Mean percentage of improvement regarding the mean RMSE reached by the worst competitor after 100 executions for the 60 datasets. 
Table 3

Correspondence between algorithms and columns for Figs. 3-6.

\begin{tabular}{ll}
\hline Column & Algorithm \\
\hline$\# 1$ & MEAN-LM \\
$\# 2$ & MED-LM \\
$\# 3$ & LM \\
$\# 4$ & MDL-LM \\
$\# 5$ & RLM \\
$\# 6$ & kNN-LM \\
$\# 7$ & WkNN-LM \\
$\# 8$ & LWLR \\
$\# 9$ & MLR \\
$\# 10$ & IBk \\
$\# 11$ & SMO-p \\
$\# 12$ & SMO-g \\
$\# 13$ & M5P \\
$\# 14$ & GP-g \\
$\# 15$ & GP-p \\
$\# 16$ & RF \\
$\# 17$ & MLP \\
\hline
\end{tabular}

Table 4

Averaged best and mean $R$ for the 60 datasets after 100 executions. The best values in bold.

\begin{tabular}{lll}
\hline Algorithm & Maximum $R$ & Mean $R$ \\
\hline MEAN-LM & 0.717 & 0.711 \\
MED-LM & 0.716 & 0.711 \\
LM & 0.785 & 0.780 \\
MDL-LM & 0.786 & 0.781 \\
RLM & 0.796 & 0.791 \\
kNN-LM & 0.765 & 0.699 \\
WkNN-LM & 0.768 & 0.696 \\
LWLR & 0.687 & 0.562 \\
MLR & 0.916 & 0.913 \\
IBk & 0.959 & 0.845 \\
SMO-p & 0.976 & 0.944 \\
SMO-g & $\mathbf{0 . 9 7 9}$ & $\mathbf{0 . 9 7 4}$ \\
M5P & 0.891 & 0.891 \\
GP-g & 0.946 & 0.851 \\
GP-p & 0.970 & 0.929 \\
RF & 0.906 & 0.898 \\
MLP & 0.969 & 0.711 \\
\hline
\end{tabular}

\subsection{Statistical analysis}

After the generation of the quality results for the different models, a statistical analysis was applied by using the open-source platform StatService [33] to check the significance in the differences among multiple methods in terms of $R$. ANOVA is usually used for multiple comparison of results if parametric conditions (homoscedasticity, independence, normality) are met [22]. However, Levene test rejected the homoscedasticity hypothesis of the results with a $p$-value under 0.001 for an $\alpha=0.05$, and therefore, a non-parametric procedure was selected. This procedure, firstly, obtained the average ranks taking into account the position of the compared results with respect to each other. Thus, a value of 1 for a rank would mean a method was the best for a test case, while a rank of $n$ would mean that it was the worst of the $n$ compared methods. Finally, the chosen procedure used the Friedman test and the Holm post hoc procedure (see [34] for a complete description of both non-parametric methods) to statistically validate the differences in the mean ranks.

Table 6 summarizes the rankings obtained for maximum $R$ and mean $R$ when Friedman's test was applied. The statistics for Friedman with control method were 815.17 and 854.02, all of them distributed according to a chi-square distribution with 16 degrees of freedom. The $p$-value for the Friedman test were less than 0.0001 so it rejected the null hypothesis (all the techniques behave in a similar way) with a level of significance of $\alpha=0.05$. Then, the Holm post hoc procedure was applied.

Table 7 shows the $p$-values, $z$-value and $\alpha$, using SMO-g as control algorithm since it obtained the best ranking in terms of maximum $R$. Note that Holm procedure rejects all the algorithm except for SMO-p, whose $\alpha$ value was greater than the required by Holm. In other words, differences between kernels applied to SVM did not provide results statistically different, which was expected, given that both of them were SVMs and the similar results they had obtained on average and that had been previously reported in Table 4.

The same information can be found in Table 8 using again SMO-g as control algorithm since it also obtained the best ranking in terms of mean $R$. As can be seen, the null hypothesis is rejected for the rest of the 16 algorithms, thus concluding that SMO-g generated the best results and they were statistically different from those of the other algorithms.

\subsection{Discussion}

Through the analysis of the results of the experimentation, it was possible to draw some interesting findings.

Regarding the attribute selection technique used, we may observe that there is a pattern in the selected set. This pattern is consistent

Table 5

Averaged improvement regarding maximum and mean RMSE obtained by the worst regressor for each of the 60 datasets after 100 executions. The best values in bold.

\begin{tabular}{lll}
\hline Algorithm & \% Maximum RMSE & \% Mean RMSE \\
\hline MEAN-LM & 19.811 & 48.737 \\
MED-LM & 20.015 & 48.726 \\
LM & 28.163 & 40.740 \\
MDL-LM & 27.458 & 40.487 \\
RLM & 30.207 & 50.106 \\
kNN-LM & 23.443 & 47.409 \\
WkNN-LM & 23.373 & 46.670 \\
LWLR & 4.517 & 25.344 \\
MLR & 57.019 & 73.572 \\
IBk & 78.055 & 61.647 \\
SMO-p & 86.751 & 84.174 \\
SMO-g & $\mathbf{9 1 . 2 2 4}$ & $\mathbf{9 1 . 9 8 1}$ \\
M5P & 51.769 & 70.443 \\
GP-g & 55.472 & 42.376 \\
GP-p & 77.752 & 62.911 \\
RF & 51.703 & 69.255 \\
MLP & 70.682 & 29.988 \\
\hline
\end{tabular}

Table 6

Rankings from maximum and mean $R$ for the 17 algorithms after 100 executions. The best in bold.

\begin{tabular}{lll}
\hline Algorithm & Maximum $R$ & Mean $R$ \\
\hline MEAN-LM & 15.408 & 13.658 \\
MED-LM & 15.433 & 13.717 \\
LM & 12.175 & 10.325 \\
MDL-LM & 12.025 & 10.258 \\
RLM & 11.258 & 9.625 \\
kNN-LM & 12.900 & 14.167 \\
WkNN-LM & 12.967 & 14.167 \\
LWLR & 15.300 & 16.233 \\
MLR & 7.533 & 4.783 \\
IBk & 3.975 & 8.117 \\
SMO-p & 2.533 & 2.400 \\
SMO-g & $\mathbf{1 . 4 5 0}$ & $\mathbf{1 . 2 0 0}$ \\
M5P & 8.467 & 5.283 \\
GP-p & 3.883 & 4.133 \\
GP-g & 5.542 & 7.733 \\
RF & 7.450 & 4.683 \\
MLP & 4.700 & 12.517 \\
\hline
\end{tabular}


Table 7

Post hoc analysis using Holm test and SMO-g as the control algorithm, in terms of maximum $R$.

\begin{tabular}{lclll}
\hline Algorithm & $p$ & $z$ & Holm & $H_{0}$ rejected \\
\hline MEAN-LM & 0 & 15.1399 & 0.0033 & $\sqrt{ }$ \\
MED-LM & 0 & 15.1671 & 0.0031 & $\sqrt{ }$ \\
LM & 0 & 11.6329 & 0.0045 & $\sqrt{ }$ \\
MDL-LM & 0 & 11.4702 & 0.005 & $\sqrt{ }$ \\
RLM & 0 & 10.6386 & 0.0056 & $\sqrt{ }$ \\
kNN-LM & 0 & 12.4193 & 0.0042 & $\sqrt{ }$ \\
WkNN-LM & 0 & 12.4916 & 0.0038 & $\sqrt{ }$ \\
LWLR & 0 & 15.0224 & 0.0036 & $\sqrt{ }$ \\
MLR & 0 & 6.5983 & 0.0071 & $\sqrt{ }$ \\
IBk & 0.0062 & 2.7387 & 0.0167 & $\sqrt{ }$ \\
SMO-p & 0.24 & 1.175 & 0.05 & \\
GP-p & 0.0083 & 2.6393 & 0.025 & $\sqrt{ }$ \\
GP-g & 0 & 4.438 & 0.01 & $\sqrt{ }$ \\
M5P & 0 & 7.6106 & 0.0063 & $\sqrt{ }$ \\
MLP & 0.0004 & 3.5251 & 0.0125 & $\sqrt{ }$ \\
RF & 0 & 6.5079 & 0.0083 & $\sqrt{ }$ \\
\hline
\end{tabular}

Table 8

Post hoc analysis using Holm test and SMO-g as control algorithm, in terms of mean $R$.

\begin{tabular}{lccll}
\hline Algorithm & $p$ & $z$ & Holm & $H_{0}$ rejected \\
\hline MEAN-LM & 0 & 13.513 & 0.0042 & $\sqrt{ }$ \\
MED-LM & 0 & 13.5762 & 0.0038 & $\sqrt{ }$ \\
LM & 0 & 9.8975 & 0.005 & $\sqrt{ }$ \\
MDL-LM & 0 & 9.8251 & 0.0056 & $\sqrt{ }$ \\
RLM & 0 & 9.1382 & 0.0063 & $\sqrt{ }$ \\
kNN-LM & 0 & 14.0643 & 0.0033 & $\sqrt{ }$ \\
WkNN-LM & 0 & 14.0643 & 0.0036 & $\sqrt{ }$ \\
LWLR & 0 & 16.3059 & 0.0031 & $\sqrt{ }$ \\
MLR & 0.0001 & 3.8867 & 0.0125 & $\sqrt{ }$ \\
IBk & 0 & 7.5022 & 0.0071 & $\sqrt{ }$ \\
SMO-p & 0.1931 & 1.3016 & 0.05 & $\sqrt{ }$ \\
M5P & 0 & 4.429 & 0.01 & $\sqrt{ }$ \\
MLP & 0 & 12.2746 & 0.0045 & $\sqrt{ }$ \\
GP-p & 0.0015 & 3.1816 & 0.025 & $\sqrt{ }$ \\
GP-g & 0 & 7.0864 & 0.0083 & $\sqrt{ }$ \\
RF & 0.0002 & 3.7782 & 0.0167 & $\sqrt{ }$ \\
& & & &
\end{tabular}

with that seen in other studies regarding the prevalence of variables related to heights (specially high percentiles) as key elements to extract knowledge from LiDAR [20]. The contribution of the intensity is mostly based on the use of minimum intensity, skewness and kurtosis. The number of returns was also a key factor to consider according to the output of the CFS method in certain databases. Selection of height percentiles is not novel but the fact that intensity variables appeared should be considered. In any case, the variables related to the intensity that were selected seem to be those most resistant to the effect of multiple returns (well-known issue in the literature [35]) since they work with the shape of their distributions (skewness, kurtosis) rather than specific values. In any case, most of the techniques used in this study had its own variable selection and the best performance (with or without previous CSF) was selected to establish the comparison with the rest of the competitors so that the importance of CFS selection should be relatively taken into account.

If we focus on the performance of the regression techniques and as can be inferred from the analysis of Fig. 3 and the subsequent statistical analysis, SMO-g (column \#13) obtained the best results regarding $R$, with an averaged maximum value of 0.979 . Moreover, from all the 100 executions with different configuration setups, it reached an $R$ of 0.974 on average. In addition, most of the rest of the studied machine learning techniques obtained high $R$ values. SMO-p even obtained non-statistically different results in terms of maximum. This involves that parameterization was adequate although, in some cases, this point can be difficult to deal with. Specially difficult was the parameterization of MLP (as can be seen comparing maximum and mean values) which suffered from a higher number of parameters than the rest which makes it harder to optimize. On the other hand, regression trees (including their ensembles, RF), which are easier to parameterize, did not get as good results showing a limited prediction power. Among them, our results show that M5P obtained the best results and its use in ensembles (RFs) increase the quality of its results even though they could not compete against SVR.

The results in terms of RMSE (Figs. 5 and 6, Table 5) also outline the previous finding. Light differences were found between the use of $R$ and RMSE for validation. This may be explained by the fact that RMSE is very sensitive to extreme values. This issue is wellknown in the literature [36] and in an environment in which databases are very small (in our case, less than 60 instances), the LOOCV procedure makes certain instances very difficult to deal with. This situation specially affects those algorithms prone to overfitting though potentially more powerful. By contrast, regression trees seem to be more resistant to overfitting although they globally do not fit as well as other techniques such as SVMs or GPs.

Finally, the most important point of this study was maybe that SVMs and GP-p outperformed MLR results according to the ranking obtained in Table 6 . This fact concurs with that previously reported $[11,12,17,19,20]$ and shows machine learning as an important tool which must be taken into account for LiDAR-derived estimation of forest variables such as the ones studied in this work.

\section{Conclusions}

This paper presented a comparison between common regression techniques in machine learning (neural networks, support vector machines, nearest neighbour, Gaussian processes, several types of regression trees, and ensembles such as Random Forest) and the classic MLR-based methodology for the estimation of forest variables from LiDAR data. The selected techniques were applied to real LiDAR data from two areas in the province of Lugo (Galizia, Spain). The results showed that SVMs statistically outperformed the rest of the techniques. Nevertheless, results confirmed recent bibliography since machine learning techniques (SVMs, GPs) obtained better results than those of classic MLR.

Future work should address gaps not covered in this work. Thus, we must complete the framework with an ad hoc feature selection for each specific method specially for those techniques that do not have it in their own building. For those techniques CFS could obtain a non-optimal subset of features when comparing with an ad hoc feature selection specially designed for them. In the same line, automatic parameterization could make the use of the most powerful techniques available for the general LiDAR users. Both problems can be solved at the same time with the application of evolutionary computation although a trade-off between optimization and run time should be reached for industrial use.

\section{Acknowledgements}

The authors would like to thank Spanish Ministry of Science and Technology, Junta de Andaluca and Pablo de Olavide University for the support under projects TIN2011-28956-C02, P12TIC-1728 and APPB813097, respectively. 


\section{References}

[1] L. Goncalves-Seco, D. Miranda, R. Crecente, J. Farto, Digital terrain model generation using airborne LIDAR in forested area of Galicia, Spain, Lisbon, Portugal, in: Proceedings of 7th International Symposium on Spatial Accuracy Assessment in Natural Resources and Environmental Sciences, 2006, pp. 169180 .

[2] W.L. Lu, K.P. Murphy, J.J. Little, A. Sheffer, H. Fu, A hybrid conditional random field for estimating the underlying ground surface from airborne lidar data, IEEE Trans. Geosci. Remote Sens. 47 (8/2) (2009) 2913-2922.

[3] M. Garcia, D. Riano, E. Chuvieco, F.M. Danson, Estimating biomass carbon stocks for a Mediterranean forest in Central Spain using LiDAR height and intensity data, Remote Sens. Environ. 114 (4) (2010) 816-830.

[4] R. Pino-Mejias, M.D.C. de-la Vega, M. Anaya-Romero, A. Pascual-Acosta, A. Jordan-Lopez, N. Bellinfante-Crocci, Predicting the potential habitat of oaks with data mining models and the R system, Environ. Model. Softw. 25 (7) (2010) 826-836.

[5] M. Mutlu, S.C. Popescu, C. Stripling, T. Spencer, Mapping surface fuel models using LiDAR and multispectral data fusion for fire behavior, Remote Sens. Environ. 112 (1) (2008) 274-285.

[6] E. Gonzalez-Ferreiro, U. Dieguez-Aranda, L. Gonçalves-Seco, R. Crecente, D. Miranda, Estimation of biomass in Eucalyptus globulus labill. forests using different LiDAR sampling densities, in: Proceedings of ForestSat, 2010.

[7] T.R. Tooke, N.C. Coops, J. Webster, Predicting building ages from LiDAR data with random forests for building energy modeling, Energy Build. 68 (Part A) (2014) 603-610.

[8] J.D. Muss, D.J. Mladenoff, P.A. Townsend, A pseudo-waveform technique to assess forest structure using discrete LiDAR data, Remote Sens. Environ. 115 (3) (2010) 824-835.

[9] J. Osborne, E. Waters, Four assumptions of multiple regression that researchers should always test, Pract. Assess. Res. Eval. 8 (2) (2002).

[10] C. Salas, L. Ene, T.G. Gregoire, E. Næsset, T. Gobakken, Modelling tree diameter from airborne laser scanning derived variables: a comparison of spatial statistical models, Remote Sens. Environ. 114 (6) (2010) 1277-1285.

[11] G. Chen, G.J. Hay, A support vector regression approach to estimate forest biophysical parameters at the object level using airborne lidar transects and quickbird data, Photogramm. Eng. Remote Sens. 77 (7) (2011) 733-741.

[12] N.R. Jachowski, M.S. Quak, D.A. Friess, D. Duangnamon, E.L. Webb, A.D. Ziegler, Mangrove biomass estimation in Southwest Thailand using machine learning, Appl. Geogr. 45 (0) (2013) 311-321.

[13] H. Latifi, A. Nothdurft, B. Koch, Non-parametric prediction and mapping of standing timber volume and biomass in a temperate forest: application of multiple optical LiDAR derived predictors, Forestry 83 (4) (2010) 395-407.

[14] J. Zhang, S. Huang, E.H. Hogg, V. Lieffers, Y. Qin, F. He, Estimating spatial variation in Alberta forest biomass from a combination of forest inventory and remote sensing data, Biogeosci. Discuss. 10 (12) (2013) 19005-19044.

[15] C.L. Wei, et al., Global patterns and predictions of seafloor biomass using random forests, PLoS ONE 5 (December (12)) (2010) e15323.

[16] J. Mascaro, et al., A tale of two forests: random forest machine learning aids tropical forest carbon mapping, PLoS ONE 9 (January (1)) (2014) e85993.

[17] K. Zhao, S. Popescu, X. Meng, Y. Pang, M. Agca, Characterizing forest canopy structure with lidar composite metrics and machine learning, Remote Sens. Environ. 115 (8) (2011) 1978-1996.

[18] A.T. Hudak, N.L. Crookston, J.S. Evans, D.E. Halls, M.J. Falkowski, Nearest neighbor imputation of species-level, plot-scale forest structure attributes from LIDAR data, Remote Sens. Environ. 112 (2008) 2232-2245.

[19] C.J. Gleason, J. Im, Forest biomass estimation from airborne LiDAR data using machine learning approaches, Remote Sens. Environ. 125 (2012) 80-91.

[20] M. Li, J. Im, L. Quackenbush, T. Liu, Forest biomass and carbon stock quantification using airborne lidar data: a case study over Huntington wildlife forest in the Adirondack park, IEEE J. Sel. Top. Appl. Earth Obs. Remote Sens., in press, 2015.

[21] D. Gagliasso, S. Hummel, H. Temesgen, A comparison of selected parametric and non-parametric imputation methods for estimating forest biomass and basal area, Open J. For. 4 (2014) 42-48.

[22] J. Demsar, Statistical comparisons of classifiers over multiple data sets, J. Mach. Learn. Res. 7 (2006) 1-30.

[23] D. Stojanova, P. Panov, V. Gjorgjioski, A. Kobler, S. Dzeroski, Estimating vegetation height and canopy cover from remotely sensed data with machine learning, Ecol. Inform. 5 (4) (2010) 256-266.

[24] L. Goncalves-Seco, E. Gonzalez-Ferreiro, U. Dieguez-Aranda, B. Fraga-Bugallo, R. Crecente, D. Miranda, Assessing attributes of high density eucalyptus globulus stands using airborne laser scanner data, Int. J. Remote Sens. 32 (24) (2011) 9821-9841.

[25] E. Gonzalez-Ferreiro, U. Dieguez-Aranda, D. Miranda, Estimation of stand variables in Pinus radiata D. Don plantations using different lidar pulse densities, Forestry 85 (2) (2012) 281-292.

[26] U. Dieguez-Aranda, et al., Herramientas selvicolas para la gestion forestal sostenible en Galicia. Xunta de Galicia, 2009.

[27] R. McGaughey, FUSION/LDV: software for LIDAR data analysis and visualization, US Department of Agriculture, Forest Service, Pacific Northwest Research Station, Seattle, 2009.

[28] A. Fakhari, A.M. Moghadam, Combination of classification and regression in decision tree for multi-labeling image annotation and retrieval, Appl. Soft Comput. 13 (2) (2013) 1292-1302.
[29] J.R. Quinlan, Learning with continuous classes, in: Proceedings of the 5th Australian Joint Conference on Artificial Intelligence, 1992, pp. 343-348.

[30] M. Robnik-Sikonja, r-cran-corelearn, 2013 〈http://mloss.org/software/view/ $310 /\rangle$.

[31] M. Hall, E. Frank, G. Holmes, B. Pfahringer, P. Reutemann, I.H. Witten, The WEKA data mining software: an update, SIGKDD Explor. 11 (1) (2009).

[32] G.F. Hughes, On the mean accuracy of statistical pattern recognizers, IEEE Trans. Inf. Theory 14 (1968) 55-63.

[33] J.A. Parejo, J. García, A. Ruiz-Cortés, J.C. Riquelme, Statservice: Herramienta de análisis estadístico como soporte para la investigación con metaheur'isticas. In: Actas del VIII Congreso Expañol sobre Metaheurísticas, Algoritmos Evolutivos y Bio-inspirados, 2012.

[34] J. Luengo, S. Garcia, F. Herrera, A study on the use of statistical tests for experimentation with neural networks: analysis of parametric test conditions and non-parametric tests, Expert Syst. Appl. 36 (2009) 7798-7808.

[35] B. Hofle, N. Pfeifer, Correction of laser scanning intensity data: data and model-driven approaches, ISPRS J. Photogramm. Remote Sens. 63 (2007) $1415-1433$.

[36] J. Tinoco, A.G. Correia, P. Cortez, Application of data mining techniques in the estimation of the uniaxial compressive strength of jet grouting columns over time, Constr. Build. Mater. 25 (3) (2011) 1257-1262. 